\title{
Frozen Section in Postmenopausal Women Presented with Suspicious Ovarian Masses, Does It Have a Role?
}

\author{
Basel Refky1', Anas Gamal'2, Emad Hamed1, Adel Fathi', Mohammad Arafa ${ }^{3}$, \\ Sameh Roshdy', Khaled Gaballa ${ }^{1}$, Essam Attia ${ }^{1}$, Mohamed Abdelkhalek ${ }^{1}$, \\ Mohamed T. Hafez ${ }^{1}$, Fayz Shahatto ${ }^{1}$, Shadi Awny', Doaa Shokry ${ }^{4}$, Gehad Tawfik ${ }^{5}$ \\ ${ }^{1}$ Surgical Oncology Unit, Oncology Center, Mansoura University, Mansoura, Egypt \\ ${ }^{2}$ Gynecology Department, Mansoura University Hospitals, Mansoura, Egypt \\ ${ }^{3}$ Pathology Department, Mansoura University, Mansoura, Egypt \\ ${ }^{4}$ Public Health Department, Mansoura Faculty of Medicine, Mansoura, Egypt \\ ${ }^{5}$ Medical Intern, Mansoura University Hospitals, Mansoura, Egypt \\ Email:dr.basel@hotmail.com
}

Received 6 November 2015; accepted 7 December 2015; published 10 December 2015

Copyright (C) 2015 by authors and Scientific Research Publishing Inc.

This work is licensed under the Creative Commons Attribution International License (CC BY).

http://creativecommons.org/licenses/by/4.0/

(c) (i) Open Access

\begin{abstract}
Background: Frozen section (FS) has a valuable role in the diagnosis of ovarian tumors. It is considered a pivotal point in guiding the surgical therapy, particularly in premenopausal women. In postmenopausal women, it may be required as well to avoid unnecessary surgical staging in benign ovarian tumors. Aim: This study aims to evaluate the accuracy of intraoperative frozen section in ovarian neoplasms in postmenopausal women. Materials and Methods: A retrospective analysis was done for intraoperative FS for suspected ovarian neoplasms. The study was conducted in Oncology Center, Mansoura University from March 2008 to December 2014. The frozen and paraffin section reports were compared, and overall accuracy, sensitivity, specificity, positive and negative predictive values were determined. Results: The study included 105 patients and the overall accuracy of FS in determining malignancy was $\mathbf{8 1 . 7 \%}$. The sensitivity for malignant tumors was $\mathbf{7 5 . 3 2 \%}$ with specificity of $\mathbf{1 0 0 \%}$. For benign tumors, the sensitivity and specificity were $\mathbf{1 0 0 \%}$ and $93 \%$, respectively. Borderline tumors had the lowest sensitivity of $100 \%$ with specificity of $\mathbf{9 5 . 1 9 \%}$. Conclusion: The present study concurs that frozen section is an accurate test for diagnosis of benign and malignant tumors in postmenopausal women thus determining the extent of surgery done for them. On the other hand, accuracy rates for borderline tumors are low.
\end{abstract}

\section{Keywords}

Frozen Section, Ovarian Cancer, Surgical Staging

How to cite this paper: Refky, B., Gamal, A., Hamed, E., Fathi, A., Arafa, M., Roshdy, S., Gaballa, K., Attia, E., Abdelkhalek, M., Hafez, M.T., Shahatto, F., Awny, S., Shokry, D. and Tawfik, G. (2015) Frozen Section in Postmenopausal Women Presented with Suspicious Ovarian Masses, Does It Have a Role? Journal of Cancer Therapy, 6, 1192-1195.

http://dx.doi.org/10.4236/jct.2015.614129 


\section{Introduction}

It is not easy to diagnose ovarian malignancy preoperatively due to avoiding of performing a preoperative biopsy for fear of capsule rupture and tumor spillage [1]. Ovarian cancers have the percentage of $4 \%$ of all cancers affecting females [2]. Nearly half of females (48\%) who are affected by malignant ovarian neoplasms are in the postmenopausal period [3]. They are considered one of the commonest causes of death among females [2] [4]. The majority of cases are diagnosed late and thus aggressive surgical management has to be performed [4]. In case of postmenopausal patients with ovarian cancer, sparing fertility is not the issue of importance but the surgical staging procedure will change according to the histopathology, for malignant ovarian tumors' complete surgical staging should be performed including omentectomy, lymphadenectomy, peritoneal biopsies and sometimes appendectomy which has got an impact on the operative time as well as morbidity and even mortality in old women. This can be avoided if we have an accurate histological diagnosis of a benign ovarian tumor pre- or intraoperative. FS is an intraoperative technique for detection pathological typing of ovarian neoplasms either benign, borderline or malignant [5].

\section{Materials and Methods}

The present study was conducted in Oncology Center, Mansoura University from March 2008 to December 2014. A retrospective analysis was performed by reviewing files of postmenopausal patients who underwent surgery as primary line of therapy for suspected ovarian neoplasms. All the frozen section diagnoses were made by a team of expert oncopathologists at the Oncology center. Rational of using frozen section is to select the appropriate management according to the staging of the tumor if benign oophorectomy and if borderline or malignant: panhystrectomy, omentectomy, iliac, para-aortic lymphadenectomy, peritonectomy and even appendectomy. The tissue sections were prepared using the Leica CM1900 cryostat (Leica microsystems, Nussloch, Germany) according to the manufacturer's instructions. Briefly, the surgical specimens were embedded in optimal cutting temperature (OCT) medium, placed on metal tissue discs which were frozen rapidly to minus $30^{\circ} \mathrm{C}$. Subsequently, they were cut frozen at about 10 micrometres thickness with the microtome portion of the cryostat. Then, picked up on glass slides and stained with hematoxylin and eosin (H\&E). The total required time from the receipt of the specimens to the examination of the tissue sections were about 20 minutes per case. The frozen section and the paraffin section reports of each patient were compared. The frozen section results were divided into the following groups: benign, borderline and malignant. The overall accuracy, sensitivity, specificity, positive and negative predictive values of the frozen section diagnoses were determined according to the status of malignancy.

\section{Statistical Analyses}

Statistical analysis was carried out using SPSS (v.20), categorical variables were presented as frequency and percentages. Agreement of the 2 variables was assessed by symmetric measure by of agreement and was measured by Spearman correlation test. P values below 0.05 were considered significant.

\section{Results}

The study included 105 patients who had undergone frozen section for ovarian masses. The mean age of the patients was 57.3 years (range 46 - 77 years). Diagnosis by frozen section was compared with paraffin section. The final histopathological diagnoses were benign in 27 patients (53.78\%), borderline in 1 (0.95\%) and malignant in 77 (42.85\%) patients. Overall accuracy of frozen section in determining the status of malignancy was $81.7 \%$. The comparison between FS diagnosis and final diagnosis shown is in Table 1. In our study, the sensitivity for benign tumors was (100\%) and the specificity was $82.05 \%$. For malignant tumors, the sensitivity and specificity were $75.32 \%$ and $100 \%$, respectively. Borderline tumors had a high sensitivity of $100 \%$ and a specificity of $95.19 \%$ but that may be due to the small number of borderline cases in our study. The positive predictive value for benign was $65.85 \%$, borderline $16.67 \%$ and malignant $100 \%$. The negative predictive value was $100 \%$, $100 \%$ and $59.57 \%$ respectively. The $\mathrm{P}$ value of chi square for benign, malignant and borderline was $<0.01 \%$, $<0.01 \%$ and $0.057 \%$, respectively. The statistical values for these three groups are listed in Table 2 .

\section{Discussion}

Preoperative biopsy in clinically suspected ovarian masses is not a part of the work up and may be contraindicated 
Table 1. Comparison between frozen section diagnosis and paraffin diagnosis.

\begin{tabular}{ccccc}
\hline & PS Benign & PS Borderline & PS Malignant & Total \\
\hline FS Benign & 27 & 0 & 14 & 41 \\
FS Borderline & 0 & 1 & 5 & 6 \\
FS Malignant & 0 & 0 & 58 & 58 \\
Total & 27 & 1 & 77 & 105 \\
\hline
\end{tabular}

Table 2. Sensitivity and specificity of frozen section in diagnosis.

\begin{tabular}{cccc}
\hline & Benign & Malignant & Borderline \\
\hline Sensitivity & $100 \%$ & $75.32 \%$ & $100 \%$ \\
Specificity & $82.05 \%$ & $100 \%$ & $95.19 \%$ \\
P value of chi square & $<0.01 \%$ & $<0.01 \%$ & $0.057 \%$ \\
Positive predictive value & $65.85 \%$ & $100 \%$ & $16.67 \%$ \\
Negative predictive value & $100 \%$ & $59.57 \%$ & $100 \%$ \\
\hline
\end{tabular}

Table 3. Sensitivity of intraoperative frozen section.

\begin{tabular}{ccccc} 
Author & Year & Benign & Borderline & Malignant \\
\hline Mohammed et al. & 2015 & $100 \%$ & $72.7 \%$ & $88.4 \%$ \\
Malipatil \& Crasta & 2013 & $99.3 \%$ & 86.66 & $84.9 \%$ \\
Suprasert et al. & 2008 & $100 \%$ & 84.2 & $92 \%$ \\
Ilvan et al. & 2005 & $100 \%$ & $87 \%$ & $87 \%$ \\
\hline
\end{tabular}

due to fear of spillage of tumor cells or capsule rupture. Staging surgery for suspected ovarian masses is a major procedure especially if extending to include omentectomy and lymphadenectomy. Intraoperative frozen section is an important procedure for diagnosis of ovarian tumors enabling the surgeon to choose the correct operation type and avoid over-treatment or under-treatment. That is very important in preserving fertility of young females. As for postmenopausal females, intraoperative frozen section procedure give the ability to avoid either overtreatment or under-treatment but in some cases under treatment can be corrected after make sure by paraffin section. The overall accuracy in our study is $81.7 \%$, this value is less than other values in previous studies which range from (90\% to 97\%) [1] [6]-[8] but still accepted. The overall accuracy which had shown in previous studies was 93.8\% [8], 95.5\% [1], 91.85\% in [9], 97.1\% [5] and this variation may be attributed to our small sample size and the raised age of cases. The sensitivity of benign, borderline and malignant ovarian tumors found $100 \%, 100 \%$ and $75.32 \%$, respectively (Table 3), and the specificity was for benign, borderline and malignant 82\%, 95.19\% and 100\%, respectively which correlate with previous studies [1] [6]-[8]. So our study recommended that FS should be done whenever possible in postmenopausal female with equivocal ovarian masses with no definite signs of malignancy either clinically, radiologically or tumor markers.

\section{Conclusion}

The present study concurs that frozen section is an accurate test for diagnosis of benign and malignant tumors in postmenopausal women thus determining the extent of surgery done for them whether limited to hysterectomy or salpingoophorectomy or extending it for complete surgical staging including panhystrectomy omentectomy, iliac and paraaortic lymphadenectomy, peritonectomy and even appendectomy. On the other hand, accuracy rates for borderline tumors are low.

\section{References}

[1] Mohammed, A.-B.F., Ahuja, V.K. and Farghaly, H. (2015) Role of Frozen Section in the Intraoperative Management 
of Ovarian Masses. Middle East Fertility Society Journal, 20, 97-101. http://dx.doi.org/10.1016/j.mefs.2014.06.001

[2] Medeiros, L.R., Rosa, D.D., Edelweiss, M.I., Stein, A.T., Bozzetti, M.C., Zelmanowicz, A. and Carballo, M.T. (2015) Accuracy of Frozen-Section Analysis in the Diagnosis of Ovarian Tumors: A Systematic Quantitative Review. International Journal of Gynecological Cancer, 15, 192-202. http://dx.doi.org/10.1111/j.1525-1438.2005.15203.X

[3] Yancik, R. (1993) Ovarian Cancer. Age Contrasts in Incidence, Histology, Disease Stage at Diagnosis, and Mortality. Cancer, 71, 517-523. http://dx.doi.org/10.1002/cncr.2820710205

[4] Subbian, A., Devi, U. and Bafna, U. (2013) Accuracy Rate of Frozen Section Studies in Ovarian Cancers: A Regional Cancer Institute Experience. Indian Journal of Cancer, 50, 302. http://dx.doi.org/10.4103/0019-509X.123599

[5] Bige, O., Demir, A., Saygili, U., Gode, F., Uslu, T. and Koyuncuoglu, M. (2011) Frozen Section Diagnoses of 578 Ovarian Tumors Made by Pathologists with and without Expertise on Gynecologic Pathology. Gynecologic Oncology, 123, 43-46. http://dx.doi.org/10.1016/j.ygyno.2011.06.030

[6] Ilvan, S., Ramazanoglu, R., Ulker Akyildiz, E., Calay, Z., Bese, T. and Oruc, N. (2005) The Accuracy of Frozen Section (Intraoperative Consultation) in the Diagnosis of Ovarian Masses. Gynecologic Oncology, 97, 395-399. http://dx.doi.org/10.1016/j.ygyno.2005.01.037

[7] Malipatil, R. and Crasta, J.A. (2013) How Accurate Is Intraoperative Frozen Section in the Diagnosis of Ovarian Tumors. Journal of Obstetrics and Gynaecology Research, 39, 710-713. http://dx.doi.org/10.1111/j.1447-0756.2012.02039.x

[8] Suprasert, P., Khunamornpong, S., Phusong, A., Settakorn, J. and Siriaungkul, S. (2008) Accuracy of Intra-Operative Frozen Sections in the Diagnosis of Ovarian Masses. Asian Pacific Journal of Cancer Prevention, 9, 737-740.

[9] Sukumaran, R., Somanathan, T., Mathews, A., Kattor, J., Sambasivan, S. and Nair, R.P. (2014) Role of Frozen Section in Intraoperative Assessment of Ovarian Masses: A Tertiary Oncology Center Experience. Indian Journal of Surgical Oncology, 5, 99-103. http://dx.doi.org/10.1007/s13193-014-0311-x 